\title{
Quasinormal modes for single horizon black holes in generic 2-d dilaton gravity
}

\author{
Joanne Kettner ${ }^{1}$, Gabor Kunstatter ${ }^{1}$ and A.J.M. Medved ${ }^{2}$ \\ 1 Physics Department \\ University of Winnipeg \\ Winnipeg, Canada R3B-2E9 \\ E-mail: g.kunstatter@uwinnipeg.ca \\ and \\ ${ }^{2}$ School of Mathematical and Computing Sciences \\ Victoria University of Wellington \\ PO Box 600, Wellington, New Zealand \\ E-Mail: joey.medved@mcs.vuw.ac.nz
}

\begin{abstract}
There has been some recent speculation that a connection may exist between the quasinormal-mode spectra of highly damped black holes and the fundamental theory of quantum gravity. This notion follows from a conjecture by Hod that the real part of the highly damped mode frequencies can be used to calibrate the semi-classical level spacing in the black hole quantum area spectrum. However, even if the level spacing can be fixed in this manner, it still remains unclear whether this implies a physically significant "duality" or merely a numerical coincidence. This tapestry of ideas serves as the motivation for the current paper. We utilize the "monodromy approach" to calculate the quasinormal-mode spectra for a generic class of black holes in two-dimensional dilatonic gravity. Our results agree with the prior literature whenever a direct comparison is possible and provide the analysis of a much more diverse class of black hole models than previously considered.
\end{abstract}

\section{INTRODUCTION}

There has been a longstanding belief that the fundamental theory of quantum gravity - whatever that might be — should be able to resolve the theoretical problems of black hole physics. Most notoriously, do black holes lose information [1], what are the microstates responsible for the (Bekenstein-Hawking) black hole entropy $[2,3]$, and should we worry about the apparent "trans-Planckian" effects of Hawking radiation [3]? (For some recent discussion on these issues, see, respectively, [4-6].) Turned around, it seems reasonable to suggest that black holes might be able to tell us something about quantum gravity. Indeed,

it has become popular to look for "imprints" from the fundamental theory at the level of classical (or semi-classical) black hole physics. For example, it has been argued - on the 
basis of black hole thought experiments - that there should be a fundamental limit on information storage capacity; with this limit coming in the guise of various entropy bounds $[7-9]$.

A similar idea, philosophically speaking, is the "Hod conjecture" [10], which relates certain quantum (spectral) features of a black hole to the quasinormal modes of its classical perturbations. In particular, Hod has suggested that the highly damped quasinormal modes correspond to fundamental vibrational modes of a black hole horizon. As such, the Bohr correspondence principle then implies that the vibrational frequencies associated with these modes must appear as transition frequencies in the semi-classical energy spectrum of a quantized black hole. As this conjecture is a central motivation for the current work, let us now elaborate on the conceptual framework.

First of all, the term "quasinormal modes" means, in a general sense, a discrete set of complex-frequency modes that are associated with any damped physical system and can be identified with complex poles in the scattering amplitude for the system under consideration. For a black hole system in particular, ${ }^{1}$ small perturbations arising near the horizon will give rise to quasinormal modes on account of the spacetime curvature acting like a damping potential. Moreover, these quasinormal modes are expected to dominate the gravitational wave signal associated with the dynamics of astrophysical black holes.

Given a black hole in an asymptotically flat spacetime, it has been demonstrated that any perturbation will generate an infinite set of such modes [12] with a spectrum that is roughly of the form

$$
\omega=\text { in }[\text { real number }]+[\text { complex number }] \text {. }
$$

Here, $n=0,1,2, \ldots$ is the discrete parameter labeling the modes, ${ }^{2}$ and the unspecified numbers depend, in general, on both the type of perturbation (primarily, on its spin and angular momentum) and the black hole background.

Now specializing to the case of a highly damped scalar or gravitational perturbation of a Schwarzschild black hole, one finds that, as $n \rightarrow \infty,{ }^{3}$

$$
\omega=i \kappa\left[n+\frac{1}{2}\right]+\frac{\kappa}{2 \pi} \ln (3)+\mathcal{O}\left[n^{-1 / 2}\right]
$$

where $\kappa$ is the (black hole) surface gravity. Note that this result has been substantiated both numerically $[13,14]$ and analytically [15-17], and also applies to $d$-dimensional Schwarzschild black holes (with $d \geq 4$ ) $[16,18,19]$. Further note that the angular momentum of the perturbation makes no contribution to the finite-order terms.

\footnotetext{
${ }^{1}$ For an comprehensive review article on the quasinormal modes of black holes, see [11].

${ }^{2}$ Note that $n$, although discrete, is not a quantum number per se.

${ }^{3}$ The relaxation time of the perturbation goes as the inverse of the imaginary part of the frequency. Hence, the limit of high damping is synonymous with the limit of large $n$. Further note that, although the highly damped limit is of interest to the theoretical community, it is actually the small $n$ modes which are most significant to gravitational wave astronomers [11].
} 
Another essential ingredient in Hod's conjecture is Bekenstein's notion of a quantized black hole area spectrum [20]. To elaborate, Bekenstein has argued — primarily, on the basis of the horizon area being an adiabatic invariant [2] — that the area $(A)$ should have a quantum spectrum of the form (in Planck units)

$$
A(m)=m \epsilon \quad \text { where } \quad m=0,1,2, \ldots,
$$

with $m$ designating the quantum number and $\epsilon$ being an undetermined constant of the order unity. ${ }^{4}$ Assuming that this is correct, one might expect the precise value of $\epsilon$ to be a "residue" of the underlying fundamental theory. In particular, as originally noted by Bekenstein and Mukhanov [22], the Bekenstein-Hawking entropy formula $(S=A / 4$ in Planck units) combined with a statistical interpretation of the entropy requires that $e^{A / 4}$ is an integer. This, in turn, constrains the spacing to take the form $\epsilon=4 \ln (k)$ for some integer $k$.

The Hod conjecture is based on the idea that the quasinormal modes - a purely classical effect - can be used to fix, unambiguously, the "quantum-gravity parameter" $\epsilon$. Hod's main point is that, "in the spirit of the Bohr correspondence principle", the real part of the highly damped mode frequencies should be interpreted as a characteristic transition frequency (say, $\omega_{c}$ ) for the black hole [10]; so that (for Schwarzschild anyways) $\omega_{c}=(\kappa / 2 \pi) \ln (3)$. Given this identification, some simple algebra then fixes $\epsilon=4 \ln (3)$. The "miracle" is that this spacing, arrived at by purely semi-classical arguments, agrees with the above constraint (as motivated by quantum gravity) and fixes $k=3$.

Remarkably, the above argument carries through, unfettered, for higher-dimensional Schwarzschild black holes (see [23] and [15]). It is perhaps useful for the ensuing discussion to show how Hod's conjecture can be applied to generic Schwarzschild-like black holes. Let us assume that there is only a single independent dimensionful parameter needed to describe the black hole geometry, which can be taken without loss of generality to be the Hawking temperature $T$. Then, on dimensional grounds, the real part of the large-damping quasinormal-mode frequency must be proportional to $T$ :

$$
\omega_{c}=\frac{\epsilon}{4} T .
$$

If $\omega_{c}$ is indeed a fundamental vibration frequency associated with black holes, then standard arguments (see [23] and references therein) imply that the following is an adiabatic invariant with an equally spaced spectrum at the semi-classical level:

$$
\int \frac{d E}{\omega_{c}}=\frac{4}{\epsilon} \int \frac{d E}{T}=\frac{4}{\epsilon} S \approx m
$$

where $E$ is the black hole energy and the thermodynamic first law has been applied. Thus, the semi-classical spacing of the Bekenstein-Hawking entropy (and, hence, the black hole area) is generically determined by $\omega_{c}$ to be

\footnotetext{
${ }^{4}$ There has since been many studies that have argued for this spectral form using more rigorous methods. See [21] for a list of references.
} 


$$
S \approx \frac{m \epsilon}{4}
$$

Given that $S$ has a statistical-mechanical interpretation in terms of black hole microstates, then $S=m \ln (k)$, which requires $\epsilon=4 \ln (k)$. Note that this argument requires only three things: (i) Hod's conjecture that $\omega_{c}$ is a fundamental oscillation frequency associated with the black hole, (ii) the first law of black hole thermodynamics, and (iii) the existence of a only a single dimensionful parameter. It is the absence of this last condition that makes it difficult to apply the above argument directly to more general types of black holes; such as black holes that are rotating or charged, or those in a non-asymptotically flat spacetime.

We should note, in passing, that the Hod conjecture has played a recent, prominent role in attempts to fix the Immirzi parameter [24] (which is closely related to Bekenstein's $\epsilon$ ) in the area spectrum of loop quantum gravity (LQG) [25]. Most significantly, it has been suggested that the presence of $\ln (3)$ implies a change in the LQG gauge group from $S U(2)$ to $S O(3)[26]$. On the other hand, some very recent calculations of the Immirzi parameter $[27,28]$ cast serious doubts on the feasibility of applying Hod's conjecture in this particular context. As this affair remains currently unsettled [29], we will comment no further on the LQG connection at this time.

It is not our intent to debate, one way or the other, whether the Hod conjecture truly represents a duality between quasinormal modes and quantum gravity or is, rather, just a numerical coincidence. (For some discussion in support of the latter viewpoint, see [30,31].) Nonetheless, one can reasonably argue that a minimal requirement for the former perspective is some degree of universality; inasmuch as $\epsilon$ should not be a particularly model-specific parameter if truly representative of the fundamental theory. Hence, one might hope that, at the very least, the same value of $\epsilon$ would be found for any single-horizon black hole in an asymptotically flat spacetime. ${ }^{5}$ If this were indeed the case, then - by virtue of the conjectured duality - one would expect the quasinormal spectra of all such black holes to limit to the same value of $\Re(\omega) / \kappa .{ }^{6}$ Thus, in order to understand the physical significance (if any) of this circle of ideas, it becomes important that the quasinormal modes are investigated in as broad a context as possible. Unfortunately, the various technical difficulties associated with quasinormal-mode calculations have impeded progress along such general lines. ${ }^{7}$

Let us now turn our attention to two-dimensional theories of dilatonic gravity. Two-

\footnotetext{
${ }^{5}$ It is now well known that, for a black hole in an asymptotically de Sitter or anti-de Sitter spacetime, the quasinormal-mode spectrum will be qualitatively much different than that of an asymptotically flat spacetime [32]. It is up to the individual reader to decide if the Hod conjecture should be required to persist under such conditions.

${ }^{6}$ One could legitimately argue that $\epsilon$ could still be dependent on the dimensionality $(d)$ without jeopardizing the conjectured correspondence. Such a dependence, however, does not occur for (at least) $d \geq 4$, since $\epsilon$ has already been calculated to be $4 \ln (3)$ for $d$-dimensional Schwarzschild black holes $[16,18,19]$.

${ }^{7}$ However, for some substantial progress at generality, see [33], which extends the WKB analysis of $[17]$.
} 
dimensional models have, often in the past, served as a useful framework for exploring gravity-related issues; both at the semiclassical and quantum level. (For reviews and references, see [34,35].) The aim of the current paper is to use this lower-dimensional context as a means for calculating the quasinormal-mode spectra for a wide class of single-horizon black holes. (Dilatonic gravity can also be used, in principle, to study more complicated types of black holes, but we will defer such endeavors until a later time.) Let us point out that, although two-dimensional theories of gravity are interesting in their own right, such models can also have physical significance as representing reduced forms of higher-dimensional gravity. For instance, a class of dilaton-gravity models arises from the spherically symmetric reduction of $d$-dimensional Einstein gravity (with $d \geq 4$ ) [36], while another class arises from the axial reduction [37] of the $(d=3)$ BTZ black hole [38].

The rest of the paper proceeds as follows. In the next section, we introduce our generic dilaton-gravity model; discussing the action, solutions and (briefly) the thermodynamics. In Section III, we consider an appropriate wave equation and cast it into a form that is suitable for the analysis of the quasinormal modes. The quasinormal spectrum is then calculated (for a still quite general class of "power-law" theories) in Section IV. More specifically, we are able to extend the powerful monodromy methodology of [16] into this dilaton-gravity framework. Section V contains a summary and discussion of our results. Finally, there is an appendix, where we provide a more detailed account of the monodromy calculation.

\section{THE MODEL}

Let us begin by introducing our generic model of interest. To be precise, we will consider the most general 1+1-dimensional action that depends on a metric tensor and a dilaton

scalar $(\Phi)$, and complies with diffeomorphism invariance and no more than two derivatives of the fields; that is $[39,40]$,

$$
I=\frac{1}{2 G} \int d^{2} x \sqrt{-\bar{g}}\left[D(\Phi) R(\bar{g})+\frac{1}{2}(\nabla \Phi)^{2}+\frac{V_{\Phi}(\Phi)}{l^{2}}\right] .
$$

Here, $D$ and $V_{\Phi}$ are arbitrary (i.e., model-dependent) functions of the dilaton, while $G$ (a dimensionless gravitational coupling) and $l$ (some length scale) may be regarded as fundamental parameters of the two-dimensional theory.

With the modest constraints that both $D(\Phi)$ and its derivative are non-vanishing functions, there exists a field reparametrization which conveniently eliminates the kinetic term of the action [41]. To elaborate, by defining

$$
\begin{gathered}
\Omega^{2}(\Phi) \equiv \exp \left[\frac{1}{2} \int \frac{d \Phi}{(d D / d \Phi)}\right] \\
g_{\mu \nu} \equiv \Omega^{2}(\Phi) \bar{g}_{\mu \nu} \\
\phi \equiv D(\Phi)
\end{gathered}
$$




$$
V_{\phi}[\phi(\Phi)] \equiv \frac{V_{\Phi}(\Phi)}{\Omega^{2}(\Phi)}
$$

we then obtain

$$
I=\frac{1}{2 G} \int d^{2} x \sqrt{-g}\left[\phi R(g)+\frac{V_{\phi}(\phi)}{l^{2}}\right] .
$$

Given that the model admits black hole solutions — which will always be assumed here it can be shown that any thermodynamic property of the black hole is invariant under such a field reparametrization $[42,43]$. Hence, it will be sufficient, for current considerations, to work with the revised form of the action (12).

One of the virtues of the reparametrized action is that the general solution can readily be obtained $[42,44]$. More specifically, the gauge choice

$$
\phi=\frac{x}{l} \geq 0
$$

leads to a (static) Schwarzschild-like metric,

$$
d s^{2}=-f(x) d t^{2}+f^{-1}(x) d x^{2},
$$

where

$$
f(x) \equiv J(x)-2 l G M
$$

Here, $M \geq 0$ is a constant of integration that can be identified with the conserved mass of the black hole [45] and

$$
J[\phi(x)] \equiv \int^{\phi=x / l} V_{\phi}(\tilde{\phi}) d \tilde{\phi}
$$

with the integration constant having already been incorporated into the observable $M$.

Although a fully general treatment is (at least in principle) possible, we will often focus on a particular class of "power-law potentials"; namely,

$$
V_{\phi} \sim \phi^{-b}
$$

so that

$$
J \sim \phi^{1-b}
$$

In concentrating on the power-law class, one of our motivations is that it enables a precise prescription for the admission of black hole solutions. The relevant class of models turns out to be $-1 \leq b<+1[46]$.

Such power-law potentials can also be motivated from the perspective of "physically relevant" models; by which we mean two-dimensional gravity models that are obtained from the dimensional reduction of a higher-dimensional theory. Most notably, the spherically symmetric reduction of $n+2$-dimensional Einstein gravity (with $n \geq 2$ ) yields, after the appropriate parametrizations, a power-law potential with $b=1 / n$ [36]. In fact, many (if 
not most) of the models considered in the literature take on a power-law form [although not necessarily in compliance with equation (23) below]. Other popular theories include Jackiw-Teitelboim gravity [47] (or, equivalently, the dimensionally reduced BTZ black hole $[38,37])$ with $b=-1$, and the Weyl-rescaled CGHS model [48] with $b=0$ [49].

For future reference, we will fix (without loss of generality) the implied prefactor in equation (17) equal to $(1-b)$, so that $J=\phi^{1-b}$ and, consequently,

$$
f(x)=\left(\frac{x}{l}\right)^{1-b}-2 l G M
$$

describes the power-law metric. The black hole horizon - in general, defined by the relation $J\left(x=x_{h}\right)=2 G M l$ - is now readily located:

$$
x_{h}=l[2 l G M]^{1 / 1-b} .
$$

As a related matter of interest, any model that does admit black hole solutions has an associated temperature $(T)$, surface gravity $(\kappa)$ and entropy $(S)$, which are given by [42]

$$
\begin{gathered}
T=\frac{\kappa}{2 \pi}=\left.\frac{1}{4 \pi} \frac{d f}{d x}\right|_{x=x_{h}}=\frac{1}{4 \pi l} V_{\phi}\left(\phi_{h}\right), \\
S=\frac{2 \pi}{G} \phi_{h},
\end{gathered}
$$

where (of course) $\phi_{h}=x_{h} / l$. Note that the temperature follows from the usual GibbonsHawking prescription [50], while the entropy then follows from the first law of thermodynamics. ${ }^{8}$ It is also worth re-emphasizing that any of these thermodynamic properties is invariant under our prior field reparametrization.

One final point concerning the thermodynamics: Rewriting the entropy as $S \sim \phi_{h} \sim$ $M^{1 /(1-b)}[c f$, equations (13) and (20)], we see that, if the entropy is to grow with the mass, the bound $b<1$ is required. Moreover, similarly re-expressing the temperature as $T \sim \phi_{h}^{-b} \sim$ $M^{-b /(1-b)}$, we observe that, if the temperature (and surface gravity) is to decrease with increasing energy, the bound $b>0$ is required. (Although counter-intuitive, the resulting negative heat capacity is the normal state of affairs for a Schwarzschild black hole [50].) On this basis, we will ultimately restrict considerations to the sub-class of power-law potentials having

$$
0<b<1
$$

Furthermore, given the power-law form, this upper bound is, as previously mentioned, necessary for the existence of black hole solutions; whereas the lower bound ensures that the physically relevant solutions of the "fundamental" action (7) will be asymptotically flat.

\footnotetext{
${ }^{8}$ The entropy can also be calculated with Wald's Noether charge formalism [51], which then via the first law — provides an independent verification for interpreting $M$ as the black hole mass.
} 


\section{THE WAVE EQUATION}

Before embarking on a quasinormal-mode analysis of our dilaton-gravity model, we first require a suitable generalization of the (usual) Klein-Gordon equation. Significantly, this equation should describe the dynamics of a matter perturbation in the two-dimensional black hole background [as described, in full generality, by equations (13)-(16)]. Let us allow for an arbitrary coupling [say, $h(\phi)]$ between the dilaton and the matter perturbation, then the following can be viewed as a natural generalization:

$$
\nabla_{h}^{2} \Psi \equiv \partial_{\mu}\left[\sqrt{-g} h(\phi) g^{\mu \nu} \partial_{\nu} \Psi\right]=0
$$

Here, the perturbation field, $\Psi=\Psi(x, t)$, is taken to be a scalar (spinless) field. This choice is motivated by two-dimensional gravity being a topological field theory, and so there can be no propagating physical modes; in particular, no gravitons nor photons [52]. (Note that, as is typical, we consider the case of a massless perturbation.)

An essential feature of $\nabla_{h}^{2}$ in two dimensions is its invariance under a conformal reparametrization of the metric. This observation (along with the similar invariance of any thermodynamic property) tells us that our prior field redefinitions will in no way effect the physical spectrum of the quasinormal modes.

From a two-dimensional perspective, the coupling $h(\phi)=h(x)$ could only be decided after one has explicitly specified the theory. On the other hand, when the model has a higherdimensional pedigree, the natural choice would be the part of the (higher-dimensional) metric determinant that is "lost" in the reduction procedure. ${ }^{9}$ For instance, in spherically

symmetric Einstein gravity, one would be inclined to set $h$ equal to $\sqrt{-g^{(n+2)}}=r^{n}$, where $r$ is the (standard) Schwarzschild radial coordinate for the $n+2$-dimensional theory. As it happens, this choice translates into $h=\phi[36]$. Nonetheless, we will keep things rather general for the moment.

To obtain a operational form of wave equation, it is convenient to (i) employ a separationof-variables technique and (ii) introduce a generalized "tortoise coordinate". The first step can be realized by expressing the perturbation field as follows:

$$
\Psi(x, t)=\frac{\psi(x)}{\sqrt{h(x)}} e^{i \omega t},
$$

where $\omega$ is the frequency and the spatial wavefunction, $\psi(x)$, has been defined in a way that ensures the simplest possible wave equation. In direct analogy to Schwarzschild calculations, the second step is realized by the relation

$$
d z=\frac{d x}{f(x)},
$$

where $z$ denotes the tortoise-like (spatial) coordinate and $f(x)$ is the metric function of equation (15). Although $z$ can not be solved for in a closed form under generic circumstances,

\footnotetext{
${ }^{9}$ This perspective follows from the presumption of diffeomorphism invariance in the higherdimensional theory.
} 
we can still make some pertinent observations: $z$ must diverge at the horizon (where $f \rightarrow 0$ ) and, for a metric of the asymptotic form $f \sim x^{1-b}, z$ will go as $x^{b}$ at spatial infinity. This means that, as long as $f \sim x^{1-b}$ with $b>0$ is valid as $x \rightarrow \infty$ [which can be viewed as a generalization of the lower bound in equation $(23)^{10}$, the black hole exterior $x \in\left(x_{h}, \infty\right)$ maps into the entire real line $z \in(-\infty,+\infty)$.

Incorporating the above relations and the static solution (13)-(16) into equation (24), we eventually obtain the following Schrodinger-like form (in direct analogy to the ReggeWheeler equation [11]):

$$
\partial_{z}^{2} \psi(z)-U[z(x)] \psi(z)=-\omega^{2} \psi(z),
$$

where the "scattering potential" is given by

$$
U(x)=\frac{1}{2} \frac{f}{h}\left[f h^{\prime \prime}+f^{\prime} h^{\prime}-\frac{1}{2} \frac{f}{h}\left(h^{\prime}\right)^{2}\right],
$$

with a prime denoting a differentiation with respect to $x$.

Significantly, we have attained a one-dimensional scattering problem (mapped to the entire real line) with a "mostly positive" potential. (By "mostly positive" it is meant that the potential is positive at least in the proximity of the horizon; this being the region of the spacetime that is most relevant to the physical scattering problem [53].) Note that it is the latter feature that leads to complex-frequency solutions and, hence, the notion of quasinormal modes.

It is, of course, the scattering potential, $U(x)$, that ultimately determines the spectral features of these quasinormal modes. For future purposes, let us specialize our generic result (28) to the power-law potential; that is, we now set $V_{\phi}(\phi)=(1-b) \phi^{-b}$ or, equivalently, $f(x)=(x / l)^{1-b}-2 l G M$ (with $0<b<1$ ). As motivated below, we also set $h=\phi^{a}$ (with $a>0)$ to obtain

$$
U(x)=\frac{a f}{4}\left[(a-2) \frac{f}{x^{2}}+\frac{2}{l} \frac{V_{\phi}}{x}\right] .
$$

Regarding the matter-dilaton coupling, it may not be a priori clear as to what form $h(\phi)$ should adopt in this framework. Nevertheless, let us recall that, in the physically motivated case of spherically reduced Einstein gravity (for which $b=1 / n$ in $n+2$ dimensions), the natural choice appears to be $h=\phi$. On this basis, it would then seem reasonable to use our power-law ansatz of $h=\phi^{a}$. To keep matters as general as possible, we will regard $a$ as an arbitrary (coupling) parameter with the constraint that $a>0$; although keeping in mind that $a=1$ is a particularly well-motivated choice. (Note that $a=0$ coincides with the trivial case of a vanishing potential. Further note that $a<0$ will jeopardize the positivity of the potential in the critical near-horizon region. ${ }^{11}$ )

\footnotetext{
${ }^{10}$ Moreover, this constraint on $f$ complies with our prior notion of asymptotic flatness. See the final paragraph in Section II.

${ }^{11}$ Actually, this positivity may not be immediately apparent for $0<a<2$. Nonetheless, the sceptical reader should notice that, for this range of $a$ values, the second (intrinsically positive) term in equation (29) will inevitably win out near the horizon (where $f \rightarrow 0$ ).
} 


\section{THE QUASINORMAL MODES}

Taking equations (27) and (29) as our starting point, we will now investigate the asymptotic nature of the associated quasinormal-mode spectrum. But, before getting into specifics, some general commentary on the nature of the problem (including the boundary conditions) is in order.

Because of our physically motivated restrictions on the solution (i.e., $0<b<1$ and $a>$ 0 ), it is clear from equation (29) that the scattering potential has the following properties: (i) it is mostly positive (in the sense described above) and (ii) it decays to zero at both the horizon and asymptotic infinity. [To be explicit, the asymptotic form as $x \rightarrow \infty$ is $U \approx a(a-2 b) /\left(4 l^{2} b^{2} z^{2}\right)$ with $z \sim x^{b}$.] On the basis of these properties — reminiscent of a highly damped system - one anticipates that there will be no normalizable bound states. However, there can still be a set of discrete states with complex-valued frequencies; these being the so-called quasinormal modes. Essentially, each of these modes is identifiable with a pole in the scattering amplitude (see [11] for further background).

Since $U[z(x)]$ goes to zero at both the horizon $(z \rightarrow-\infty)$ and spatial infinity $(z \rightarrow \infty)$, it immediately follows from equation (27) that

$$
\psi(z) \sim e^{ \pm i \omega z} \quad \text { as } \quad z \rightarrow \pm \infty .
$$

One normally proceeds by imposing "radiation" boundary conditions, so that the mode is a purely ingoing plane wave at the horizon and purely outgoing at infinity. Considering our prior convention of $\Psi \sim e^{+i \omega t}$, the appropriate boundary conditions become

$$
\begin{aligned}
& \psi(z) \sim e^{+i \omega z} \quad \text { as } \quad z \rightarrow-\infty, \\
& \psi(z) \sim e^{-i \omega z} \quad \text { as } \quad z \rightarrow+\infty .
\end{aligned}
$$

Note that any damped mode must decay with time [and so $\Im(\omega)>0$ ]; meaning that the "absent components" (outgoing at the horizon and ingoing at infinity) would have to be exponentially suppressed anyways. This subtlety (amongst others) has impeded general progress in quasinormal mode calculations. One possible resolution, as proposed in [16], is to analytically continue $z$, instead of $\omega$, and then consider the boundary conditions as the product $\omega z \rightarrow \pm \infty$. Consult [16] for further discussion.

There are, by now, many documented techniques for calculating the quasinormal-mode frequencies; both numerical and analytical. (For an up-to-date survey, see [19].) Here, we will focus on an analytical method that is particularly well suited for determining the frequencies in the highly damped $[\Im(\omega) \rightarrow \infty]$ limit; namely, the "monodromy procedure" of Motl and Neitzke [16]. In a nutshell, their methodology is based (in a Schwarzschild framework) on considering the complex- $r$ plane (including the black hole interior) and then computing the monodromy that the wavefunction picks up (relevant to the singular horizon point) in two distinct ways. These two calculations can then be matched - in the limit of high damping - resulting in an equation from which the quasinormal-mode spectrum can readily be extracted. It is encouraging that, for the four-dimensional Schwarzschild black hole, their outcome agrees with earlier numerical calculations $[13,14]$. 
Although we intend to adapt this monodromy method to our generic dilaton model, it will (thankfully) not be necessary to reiterate the rather technical procedure. (We do, however, provide a somewhat technical outline of the procedure in an appendix. The interested reader can also consult the original work [16] for further details.) Instead, we will demonstrate the similarities between our problem and the Schwarzschild one, and use this as a basis for suitably generalizing the results of [16].

First of all, let us point out that the complex- $r$ plane of the Schwarzschild black hole is qualitatively similar to the complex- $x$ plane of our dilatonic black hole model. In particular, the key elements in common are two regular singular points at the spatial origin and the black hole horizon and an irregular singular point at spatial infinity. Note that the calculation is insensitive to any subtle differences that may exist. Actually, what is important to the monodromy calculation is the near-origin form of the scattering potential when expressed in terms of the tortoise coordinate; this point is elaborated on in section 3.1 of [16] (where the authors generalize considerations to higher-dimensional Schwarzschild black holes — also see $[18])$.

In view of the above observation, let us see what does happen to be the behavior of our scattering potential near the spatial origin; especially, its functional dependence on the generalized tortoise coordinate [i.e., $z$ as defined by equation (26)]. To proceed, it will be necessary for us to take the near-origin limit of a few quantities. First consider that (near $x=0) f \approx-2 l G M$. Then, since $V / x \sim x^{-(b+1)}$ "blows up" slower than $x^{-2}$, equation $(29)$ gives us

$$
U(x \sim 0) \approx \frac{a(a-2)(2 l G M)^{2}}{4 x^{2}} .
$$

Now consider that, in the near-origin limit, equation (26) can be trivially integrated to yield $x=-(2 l G M) z .{ }^{12}$ Hence, the above expression for $U$ can be rewritten as

$$
U(z \sim 0) \approx \frac{a(a-2)}{4 z^{2}}
$$

Remarkably, this is identical to the near-origin form of the potential derived in $[16][c f$, their equation (13)], insofar as we make the identification

$$
a=1 \pm j
$$

where $j$ is the spin associated with the Schwarzschild perturbation. (Note that, typically, $j=0,1,2$, etc. - so that, given $j$, we can choose the sign by requiring that $a>0$.)

In view of these observations, the monodromy calculation of [16] should carry through, almost verbatim, provided that we make the specified substitution. In this spirit, we have obtained (see the appendix for an elaboration) the following relation:

$$
e^{2 \pi \omega / \kappa}=-(1+2 \cos [\pi(a-1)])
$$

\footnotetext{
${ }^{12}$ As in [16], the constant of integration is chosen so that $z=0$ at the origin.
} 
where $\kappa$ is the surface gravity of the dilatonic black hole $[c f$, equation (21)]. It is worth emphasizing that $\kappa$ appears because of a contour integral around the event horizon. Moreover, the appearance of the surface gravity is, in fact, a universal consequence of the near-horizon form of the tortoise coordinate. By which we mean that

$$
z=\int \frac{d x}{f(x)} \approx \int \frac{d x}{\left(x-x_{h}\right) f^{\prime}\left(x_{h}\right)}=\frac{1}{2 \kappa} \ln \left(x-x_{h}\right)
$$

is universally valid. (Also see equation (47) of [16] along with the related discussion.)

Another interesting feature of the relation (36) is that the right-hand side depends only on the value of $a$; namely, a parameter that determines the coupling between the dilaton and the matter perturbation. Which is to say, the quasinormal modes seem rather indifferent to the form of the gravitational action itself; hence, we can see the first glimpses of the universality that is suggested by the Hod conjecture [10].

Although $a$ was left arbitrary in our formalism, two results immediately jump out. Firstly, when $a$ is any even integer, the right-hand side of this expression becomes unity, and it follows that the real part of each mode frequency vanishes asymptotically. This is similar to what is found for electromagnetic $(j=1)$ perturbations in many black hole models (including Schwarzschild). Meanwhile, when $a$ is any odd integer, the right-hand side becomes -3 . Solving this relation, we find that

$$
\omega=i \kappa\left[n+\frac{1}{2}\right]+\frac{\kappa}{2 \pi} \ln (3),
$$

where $n$ takes on positive integral values (the positivity is enforced by the boundary condition that $\Im(\omega)>0$ ). Actually, this is not quite right because, as previously mentioned, the monodromy calculation only has validity in the asymptotic limit of high damping. Hence, it is more accurate to write

$$
\omega=i \kappa\left[n+\frac{1}{2}\right]+\frac{\kappa}{2 \pi} \ln (3)+\mathcal{O}\left[n^{-1 / 2}\right] \quad \text { as } \quad n \rightarrow \infty .
$$

But, any way you slice it, this is precisely the highly damped spectrum that is obtained for scalar $(j=0)$ or gravitational $(j=2)$ perturbations of a $d$-dimensional Schwarzschild black hole (with $d \geq 4$ ). In particular, $a=1$ - that is, the preferred choice of coupling parameter as motivated by spherically symmetric Einstein gravity - gives us precisely the Schwarzschild spectrum. This "coincidence" provides a nice consistency check for our calculation.

\section{DISCUSSION}

In summary, we have considered a generic model of two-dimensional dilatonic gravity coupled to a (scalar) matter perturbation. Most importantly, we have utilized the monodromy technique of [16] to calculate the frequency of the highly damped quasinormal modes; including the (sub-leading) real part. For the particular case of spherically symmetric reduced

Einstein gravity, we were able to substantiate that the real part goes asymptotically to $T \ln (3)$; in compliance with previous calculations for a $d$-dimensional Schwarzschild black hole $[16,18,19]$. 
An interesting subplot is the direct connection between $a$ (a parameter which determines the two-dimensional matter-dilaton coupling) and $j$ (the spin of the higher-dimensional matter perturbation); $c f$, equation (35). Consequently, we can effectively mimic different types of perturbations by judiciously selecting the parameter $a$; even though, typically speaking, two-dimensional gravity can only support scalar $(j=0)$ perturbations. It might prove to be informative if this connection could be given a physical motivation.

Although the agreement with known results is quite gratifying, our formalism is making a much more powerful statement than just this. To elaborate, one might expect that virtually any single-horizon and asymptotically flat black hole spacetime would have a dimensionally reduced form that complies (after a suitable process of reduction and reparametrization) with the model we have been studying. Then, since the final result for the mode spectra depends only on $\kappa$ and $a$, it is feasible that, for any such black hole, the real part of the frequencies may asymptote to $T \ln (3)$. If this were indeed the case, it would establish conjectures of universality that have been made in, for instance, [23,33]. This type of universality should be regarded as a minimal requirement if one is to take the Hod conjecture [10] seriously as a statement about quantum gravity.

On the other hand (staying with the Hod conjecture), our calculation enables one to see, quite clearly, where the relevant contributions to the mode spectra come from. For instance, the exponent in equation (36) or $2 \pi \omega \kappa$ (i.e., the surface gravity) comes directly from a contour integral around the event horizon. Moreover, the $\ln (3)$ comes from the matterdilaton coupling, with the choice of $a=1$ (for spherically reduced Einstein gravity) being a direct consequence of the diffeomorphism invariance of the higher-dimensional theory. With this knowledge in hand, there no longer seems to be anything "mystical" about the presence of $\ln (3)$. In fact, it becomes difficult to see where there could be any deep connection with quantum gravity!

Irrespective of such speculations about quantum gravity, it would be interesting to see how much more can be deduced about quasinormal modes in the lower-dimensional framework of dilatonic gravity. The power of two-dimensional gravity is that it allows one to examine a large class of theories with a single and relatively simple model; with the underlying diversity being reflected in a few adjustable parameters (such as our power-law coefficient $b$ ). For starters, we hope to extend the analysis beyond this simple power-law class of potentials; part of our motivation for maintaining generality throughout much of the analysis. In this regard, it should, however, be pointed out that our quasinormal-mode analysis depends (for the most part) only on the behavior of the tortoise coordinate at the horizon, the origin and spatial infinity. Which is to say, one can readily confirm that the prior analysis carries right through as long as, for a given model, the following conditions are met: (i) $V_{\phi}$ maintains the necessary asymptotic behaviors; namely, $V_{\phi} \sim \phi^{-b}$ with $b<1$ near the origin [thus ensuring $U(\sim 0) \sim z^{-2}$ ] and $V_{\phi} \sim \phi^{-b}$ with $b>0$ at spatial infinity [thus ensuring $z \rightarrow \infty$ ], and (ii) $V_{\phi}$ is a monotonic function of $\phi$ (because, given the correct asymptotic behaviors, this would be sufficient to ensure a single-horizon black hole solution).

However, it is not so trivial to generalize matters to dilaton-gravity models with two conserved charges (or, equivalently, spacetimes with two horizons). Such models arise quite naturally when one considers, for instance, spherically reduced Einstein-Maxwell gravity $[54,55]$. Progress along these lines could certainly help us to better understand the physical meaning of the Hod conjecture and, perhaps, quantum gravity in general. 
Finally, let us end with a discussion on what is a generic issue of the monodromy procedure. At an intermediate step in the calculation, one finds that the formalism breaks down for a scalar perturbation $(j=0)$ in higher-dimensional studies [16] or for an $a=1$ coupling in ours. [See the note following equation (50).] This is, however, not really a problem, as long as one understands that the $j=0$ or $a=1$ case is to be handled in a "limiting sense" [56]. Nevertheless, one might well ask if there is some sort of physical significance to this type of occurrence.

To address this last point, let us make a pertinent observation as follows: If we redefine the spatial wavefunction so that

$$
\chi(x) \equiv \sqrt{f(x)} \psi(x),
$$

then the scattering equation (27) can be recast into the form ${ }^{13}$

$$
\chi^{\prime \prime}+R(x) \chi=0,
$$

where

$$
R(x)=\frac{1}{f^{2}}\left[\omega^{2}-U(x)+\frac{\left(f^{\prime}\right)^{2}}{4}-\frac{f f^{\prime \prime}}{2}\right] .
$$

Now specializing to our power-law model, we find that, near the origin,

$$
R(x \sim 0) \approx-\frac{a(a-2)}{4 x^{2}} .
$$

Hence, the near-origin form of the (revised) scattering equation (41) can be written as

$$
H \chi(x)=0,
$$

with the "Hamiltonian" operator being expressible as

$$
H=-\frac{1}{2} \frac{d}{d x^{2}}+\frac{g_{c}}{2 x^{2}}
$$

where we have defined a "coupling constant", $g_{c} \equiv a(a-2) / 4$. Remarkably, this is exactly the form of the Hamiltonian operator for conformal quantum mechanics [57]. ${ }^{14}$ Moreover,

\footnotetext{
${ }^{13}$ Note that this form can also be used as the basis for a WKB analysis of the quasinormal modes [17] (also see [33]). The full WKB analysis for the case of generic two-dimensional dilatonic gravity will be presented elsewhere.

${ }^{14}$ It should be noted that the same observation about conformal quantum mechanics also follows from the $z$-coordinate form of the scattering equation; $c f$, equation (34). Nonetheless, we have chosen to work in terms of the coordinate $x$ because the above formalism makes it quite clear as to how the Hamiltonian can take on the conformal form both near the origin and at the horizon; $c f$, the third term on the right-hand side of equation (42).
} 
the well-known critical value of the coupling constant $-g_{c}=-1 / 4-$ occurs when $a=1$; precisely at the same point where the limiting procedure is required.

We expect that the appearance of conformal quantum mechanics is more than a mere coincidence. Indeed, this same form of Hamiltonian appears when a scalar probe is used to investigate the near-horizon dynamics of a Schwarzschild black hole [58,59]; clearly, a dynamical framework that is closely related to the quasinormal modes. Furthermore, as speculated in [59], there is probably a deep connection between this realization of conformal mechanics and calculations of the black hole entropy that are based on near-horizon conformal symmetries $[60,61]$. We hope to readdress such fascinating ideas at a future time.

\section{APPENDIX: THE MONODROMY CALCULATION}

Here, we provide a more detailed account of how equation (36) is obtained by way of the monodromy approach. We will, however, provide only a somewhat brief sketch of the computation; glossing over many of the technical caveats. The reader is referred, once again, to [16] for many of the finer subtleties of the calculational procedure.

The basic idea is to impose the highly damped limit or $\Im(\omega)>>\Re(\omega)$ (with both quantities taken to be positive) and then calculate the monodromy of the wavefunction in two distinct ways. These two results can then be matched to obtain the desired relation.

We begin here by considering a suitably constructed contour in the complex- $x$ plane or, more accurately, the complex- $z$ plane. However, before getting started, we will (for "best results") perform a "Wick rotation" of $z$, so that the boundary condition at spatial infinity $(z$ or $x \rightarrow \infty$ ) now becomes $\omega z \rightarrow \infty$. It is convenient if the contour is initiated in this particular asymptotic region - effectively, this means starting on the negative imaginary axis of $z$ - where we know $[c f$, equation (32)] that

$$
\psi(z) \sim e^{-i \omega z} \quad \text { as } \quad \omega z \rightarrow+\infty .
$$

Next, we follow the line $\Im(\omega z)=0$ until the near-origin region is reached. (Note that the plane-wave behavior should prevail until this interior region, since $\omega^{2}$ will dominate over the scattering potential up to a small neighborhood of the origin.) Let us appropriately recall the near-origin form of the scattering equation [cf, equations (27) and (34)],

$$
\left[-\partial_{z}^{2}+\frac{a(a-2)}{4 z^{2}}-\omega^{2}\right] \psi(z)=0 \quad \text { as } \quad z \rightarrow 0 .
$$

Conveniently, this equation can be solved exactly as a linear combination of Bessel functions [62]:

$$
\psi(z)=A_{+} c_{+} \sqrt{\omega z} J_{+\nu}(\omega z)+A_{-} c_{-} \sqrt{\omega z} J_{-\nu}(\omega z),
$$

where $\nu \equiv(a-1) / 2$, while the products $c_{+} A_{+}$and $c_{-} A_{-}$represent constant coefficients. Following [16], we will choose the "normalization factors" (denoted by $c_{ \pm}$) so that

$$
c_{ \pm} \sqrt{\omega z} J_{ \pm \nu}(\omega z) \sim 2 \cos \left(\omega z-\alpha_{ \pm}\right) \quad \text { as } \quad \omega z \rightarrow \infty
$$

with 


$$
\alpha_{ \pm} \equiv \frac{\pi}{4}[1 \pm 2 \nu]=\frac{\pi}{4}[1 \pm(a-1)]
$$

Note that, when $\nu=0$, the Bessel function is simply a constant and equation (49) is, strictly speaking, no longer accurate. Hence, when applied to the case of $a=1$, the subsequent formalism should be understood in a "limiting sense" [56].

Using equations (48) and (49), as well as the boundary condition of equation (32) (which indicates that the coefficient of $e^{+i \omega z}$ must vanish as $\left.\omega z \rightarrow \infty\right)$, we can deduce the constraint

$$
A_{+} e^{-i \alpha_{+}}+A_{-} e^{-i \alpha_{-}}=0
$$

Reapplying equations (48) and (49), we also find that

$$
\psi(z) \sim\left[A_{+} e^{+i \alpha_{+}}+A_{-} e^{+i \alpha_{-}}\right] e^{-i \omega z} \quad \text { as } \omega z \rightarrow \infty .
$$

Let us now continue the contour in the following manner: We want to extend the contour from the negative imaginary axis of $z(\Im(\omega z) \rightarrow \infty)$ to the positive imaginary axis $(\Im(\omega z) \rightarrow-\infty)$ while missing the origin. As it turns out, this action necessitates a counterclockwise rotation of $3 \pi$ about the singularity $[16,18,56$. [In determining this angle, we are appealing to the structural similarity between our model and the Schwarzschild one; especially with regard to the complex- $z$ plane in the limit of high damping. More to the point, when $|\omega|^{2}$ is large enough - thereby dominating the scattering problem except in a small neighborhood of the origin - then only the near-origin form of the potential is important. Relevantly, the near-origin form of our potential is identical to that of the Schwarzschild model when each is expressed in terms of their respective tortoise coordinates. This means that, essentially, any (hypothetical) observer "living" in the complex $z$-plane would be unable to distinguish between the highly damped limits of either scattering problem. ${ }^{15}$ ] Using some well-documented transformation properties of the Bessel functions [62] to handle the rotation, we eventually arrive at

$$
c_{ \pm} \sqrt{\omega z} J_{ \pm \nu} \sim e^{6 i \alpha_{ \pm}} 2 \cos \left(-\omega z-\alpha_{ \pm}\right) \quad \text { as } \quad \omega z \rightarrow-\infty
$$

By way of equations (48) and (53), we then have

$$
\psi(z) \sim\left[A_{+} e^{5 i \alpha_{+}}+A_{-} e^{5 i \alpha_{-}}\right] e^{-i \omega z}+\left[A_{+} e^{7 i \alpha_{+}}+A_{-} e^{7 i \alpha_{-}}\right] e^{+i \omega z} \quad \text { as } \quad \omega z \rightarrow-\infty .
$$

Finally, the contour is continued (clockwise) along a large semicircle — encircling the horizon - that brings us from the asymptotic region $\omega z \rightarrow-\infty$ back to our starting point of $\omega z \rightarrow \infty$. Significantly, the semicircle is strictly in the region where $\omega^{2}$ dominates over the potential, and so plane-wave behavior again prevails. Given our limit of infinitely large damping (meaning that a WKB type of approximation for the scattering problem must certainly be valid), it follows that, after completing this contour, the coefficient of

\footnotetext{
${ }^{15}$ Note that, by dealing directly with the complex- $z$ plane, we circumvent any subtle differences that might exist between our complex- $x$ plane and the Schwarzschild complex- $r$ plane.
} 
the asymptotically dominant plane wave $\left(e^{-i \omega z}\right)$ should remain unchanged. ${ }^{16}$ Hence, this outgoing plane wave picks up the following monodromy about the specified contour $[c f$, equations (52) and (54)]:

$$
\frac{A_{+} e^{5 i \alpha_{+}}+A_{-} e^{5 i \alpha_{-}}}{A_{+} e^{i \alpha_{+}}+A_{-} e^{i \alpha_{-}}}
$$

Using the boundary constraint (51) to eliminate the constants $A_{ \pm}$and then simplifying, one finds that equation (55) reduces to

$$
\frac{e^{6 i \alpha_{+}}-e^{6 i \alpha_{-}}}{e^{2 i \alpha_{+}}-e^{2 i \alpha_{-}}}=-\frac{\sin (3 \pi \nu)}{\sin (\pi \nu)}=-[1+2 \cos (2 \pi \nu)] .
$$

The above analysis completes one way of calculating the monodromy. A second way follows from the observation that the only singularity of $\psi(z)$ inside our chosen contour occurs at the horizon. So let us consider the near-horizon form of the wavefunction $[c f$, equations (31) and (37)],

$$
\psi(z) \sim e^{+i \omega z} \sim \exp \left[i \omega \ln \left(x-x_{h}\right) /(2 \kappa)\right] \quad \text { as } \quad z \rightarrow-\infty .
$$

This form tells us that going once around the horizon singularity clockwise (i.e., our chosen contour), multiplies the wave function by $e^{2 \pi \omega /(2 \kappa)}$. However, the same action multiplies the outgoing wave $\left(e^{-i \omega z}\right)$ by $e^{-2 \pi \omega /(2 \kappa)}$. That is to say, the coefficient of $e^{-i \omega z}$ must be multiplied by a monodromy of

$$
\frac{e^{2 \pi \omega /(2 \kappa)}}{e^{-2 \pi \omega /(2 \kappa)}}=e^{2 \pi \omega / \kappa}
$$

Finally, matching the two calculations for the monodromy (56) and (58) and then inserting $\nu=(a-1) / 2$, we obtain our spectral-defining relation; namely, equation (36).

\section{ACKNOWLEDGMENTS}

Research for AJMM is supported by the Marsden Fund (c/o the New Zealand Royal Society) and by the University Research Fund (c/o Victoria University). Research for GK is supported by the Natural Sciences and Engineering Research Council of Canada.

\footnotetext{
${ }^{16}$ Conversely, the same claim can not be made about the coefficient of $e^{+i \omega z}$, which is exponentially suppressed and, therefore, much more sensitive to small corrections in a WKB-like approximation.
} 


\section{REFERENCES}

[1] S.W. Hawking, Phys. Rev. D14, 2460 (1976).

[2] J.D. Bekenstein, Lett. Nuovo. Cim. 4, 737 (1972); Phys. Rev. D7, 2333 (1973); Phys. Rev. D9, 3292 (1974).

[3] S.W. Hawking, Nature 248, 30 (1974); Comm. Math. Phys. 43, 199 (1975).

[4] S.B. Giddings and N. Lippert, Phys. Rev. D69, 124019 (2004) [hep-th/0402073].

[5] D.V. Fursaev, "Can one understand black hole entropy without knowing much about quantum gravity?", arXiv:gr-qc/0404038 (2004).

[6] A.D. Helfer, Rept. Prog. Phys. 66, 943 (2003) [gr-qc/0304042].

[7] J.D. Bekenstein, Phys. Rev. D23, 287 (1981).

[8] G. 't Hooft, "Dimensional Reduction in Quantum Gravity", arXiv:gr-qc/9310026 (1993).

[9] L. Susskind, J. Math. Phys. 36, 6377 (1995) [hep-th/9409089].

[10] S. Hod, Phys. Rev. Lett. 81, 4293 (1998) [gr-qc/9812002].

[11] K.D. Kokkotas and B.G. Schmidt, Living Rev. Rel. 2, 2 (1999) [gr-qc/9909058].

[12] A. Bachelot and A. Motet-Bachelot, "Resonances of Schwarzschild black holes", in Proceedings of the IV International Conference of Hyperbolic Problems, ed. Vieweg (Taosmina, 1992).

[13] H.-P. Nollert, Phys. Rev. D47, 5253 (1993).

[14] N. Andersson, Class. Quant. Grav. 10, L61 (1993).

[15] L. Motl, Adv. Theor. Math. Phys. 6, 1135 (2003) [gr-qc/0212096].

[16] L. Motl and A. Neitzke, Adv. Theor. Math. Phys. 7, 307 (2003) [hep-th/0301173].

[17] N. Andersson and C.J. Howls, Class. Quant. Grav. 21, 1623 (2004) [gr-qc/0307020].

[18] D. Birmingham, Phys. Lett. B569, 199 (2003) [hep-th/0306004].

[19] V. Cardoso, J.P.S. Lemos and S. Yoshida, Phys. Rev. D69, 044004 [gr-qc/0309112].

[20] J.D. Bekenstein, Lett. Nuovo Cimento 11, 467 (1974).

[21] S. Das, H. Mukhopadhyay and P. Ramadevi, "Spectrum of rotating black holes and its implications for Hawking radiation", arXiv:hep-th/0407151.

[22] J.D. Bekenstein and V.F. Mukhanov, Phys. Lett. B360, 7 (1995) [gr-qc/9505012].

[23] G. Kunstatter, Phys. Rev. Lett. 90, 161301 (2003) [gr-qc/0212014].

[24] G. Immirzi, Nucl. Phys. Proc. Suppl. 57, 65 (1997) [gr-qc/9710014].

[25] A. Ashtekar, J.C. Baez and K. Krasnov, Adv. Theo. Math. Phys. 4,1 (2000) [grqc/0005126].

[26] O. Dreyer, Phys. Rev. Lett. 90, 081301 (2003) [gr-qc/0211076].

[27] M. Domagala and J. Lewandowski, "Black hole entropy from quantum geometry", arXiv:gr-qc/0407051 (2004).

[28] K.A. Meissner, "Black hole entropy in loop quantum gravity", arXiv:gr-qc/0407052 (2004).

[29] S. Alexandrov, "On the counting of black hole states in loop quantum gravity", arXiv:grqc/0408033 (2004).

[30] A.J.M. Medved and D. Martin, "A note on quasinormal modes: A tale of two treatments", arXiv:gr-qc/0311086 (2003) and to appear in the conference proceedings for ACGR4.

[31] I.B. Khriplovich, "Quasinormal modes, quantized black holes, and correspondence principle", arXiv:gr-qc/0407111 (2004). 
[32] V. Cardoso, J. Natario and R. Schiappa, "Asymptotic quasinormal frequencies for black holes in non-asymptotically flat spacetimes", arXiv:hep-th/0403132 (2004).

[33] T. Tamaki and H. Nomura, "The universal area spectrum in single-horizon black holes", arXiv:hep-th/0405191 (2004).

[34] S. Nojiri and S.D. Odintsov, Int. J. Mod. Phys. A16, 1015 (2001) [hep-th/0009202].

[35] D. Grumiller, W. Kummer and D.V. Vassilevich, Phys. Rept. 369, 327 (2002) [hepth/0204253].

[36] G. Kunstatter, R. Petryk and S. Shelemy, Phys. Rev. D57, 3537 (1998) [gr-qc/9709043].

[37] A. Achucarro and M.E. Ortiz, Phys. Rev. D48, 3600 (1993) [hep-th/9304068].

[38] M. Banados, C. Teitelboim and J. Zanelli, Phys. Rev. Lett. 69, 1849 (1992) [hepth/9204099].

[39] T. Banks and M. O’Loughlin, Nucl. Phys. B362, 649 (1991).

[40] S.D. Odintsov and I. Shapiro, Phys. Lett. B263, 183 (1991).

[41] D. Louis-Martinez, J. Gegenberg and G. Kunstatter, Phys. Lett. B321, 193 (1994) [gr-qc/9309018].

[42] J. Gegenberg, G. Kunstatter and D. Louis-Martinez, Phys. Rev. D51, 1781 (1995) [gr-qc/9408015].

[43] M. Cadoni, Phys. Lett. B395, 10 (1997) [hep-th/9610201].

[44] T. Strobl, Poisson Structure Induced Field Theories and Models of $1+1$ Dimensional Gravity, PhD Thesis at TU-Vienna (1994) [hep-th/0011248].

[45] R.B. Mann, Phys. Rev. D47, 4438 (1993) [hep-th/9206044].

[46] M. Cadoni, Phys. Rev. D53, 4413 (1996) [gr-qc/9510012].

[47] R. Jackiw in Quantum Theory of Gravity, ed. S. Christensen (Hilger, Bristol,1984), p.403; C. Teitelboim, ibid, p.327; R. Jackiw, Nucl. Phys. B252, 343 (1985).

[48] C.G. Callan, S.B. Giddings, J.A. Harvey and A. Strominger, Phys. Rev. D45, 1005 (1992) [hep-th/9111056].

[49] M. Cadoni and S. Mignemi, Phys. Lett. B358, 217 (1995) [hep-th/9410041].

[50] G.W. Gibbons and S.W. Hawking, Phys. Rev. D15, 2752 (1977).

[51] R.M. Wald, Phys. Rev. D48, 3427 (1993) [gr-qc/9307038]; V. Iyer and R.M. Wald, Phys. Rev. D50, 846 (1994) [gr-qc/9403028].

[52] D. Birmingham, M. Blau, M. Rakowski and G. Thompson, Phys. Rept. 209, 129 (1991).

[53] A.J.M. Medved, D. Martin and M. Visser, Class. Quant. Grav. 21, 1393 (2004) [grqc/0310009].

[54] D. Louis-Martinez and G. Kunstatter, Phys. Rev. D52, 3494 (1995) [gr-qc/9503016].

[55] A.J.M. Medved and G. Kunstatter, Phys. Rev. D59, 104005 (1999) [hep-th/9811052].

[56] K. Krasnov and S.N. Solodukhin, "Effective stringy description of Schwarzschild black holes", arXiv:hep-th/0403046 (2004).

[57] V. de Alfaro, S. Fubini and G. Furlan, Nuovo. Cim. 34A, 569 (1976).

[58] T.R. Govindarajan, V. Suneeta and S. Vaidya, Nucl. Phys. B583, 291 (2000) [hepth/0002036].

[59] D. Birmingham, K.S. Gupta and S. Sen, Phys. Lett. B505, 191 (2001) [hep-th/0102051].

[60] S. Carlip, Phys. Rev. Lett. 82, 2828 (1999) [hep-th/9812013]; Class. Quant. Grav. 16, 3327 (1999) [gr-qc/9906126]; Phys. Rev. Lett. 88, 241301 [gr-qc/0203001].

[61] S.N. Solodukhin, Phys. Lett. B454, 213 (1999) [hep-th/9812056].

[62] Handbook of Mathematical Functions, ed. M. Abramowitz and I.A. Stegun (Dover Pub- 
lications, New York, 1964). 